\title{
3 A perturbação de pós-stress traumático e o sentido de coerência em mulheres com cancro da mama
}

\author{
I Ana Margarida Varela ${ }^{1}$; Isabel Leal ${ }^{2}$ _
}

\section{RESUMO}

No presente estudo investigou-se a relação entre a perturbação de pós-stress traumático e o sentido de coerência, numa amostra de 84 mulheres a quem foi diagnosticado cancro da mama. Para tal, utilizou-se um protocolo de investigação constituído pelo Questionário de Identificação, o Questionário de Orientação para a Vida - QOV (Nunes, 1999) e a PTSD Checklist Civilian Version - PCL-C (Cordova, Andrykowski, Kenady, McGrath, Sloan \& Redd, 1995). Os dados obtidos indicam que a perturbação de pós-stress traumático está associada a níveis inferiores de sentido de coerência. Podendo-se concluir que o facto de o mundo ser perspectivado como compreensível, flexível e com significado, promove a adaptação das mulheres a quem foi diagnosticado cancro da mama às contingências da doença, logo a exibição por parte destas de níveis inferiores de perturbação de pós-stress traumático.

PALAVRAS-CHAVE: Cancro da mama; perturbação de pós-stress traumático; sentido de coerência

\begin{abstract}
In the present study were investigated the posttraumatic stress disorder and the sense of coherence in a sample of 84 women with breast cancer. To this end, was developed a research protocol made of a Questionnaire of identification, the Orientation to Life Questionnaire - QOV (Nunes, 1999) and the PTSD Checklist Civilian Version - PCL-C (Cordova, Andrykowski, Kenady, McGrath, Sloan \& Redd, 1995). The data obtained showed that that postraumatic stress disorder were associated with a fewer degree of sense of coherence. We conclude that the fact that the world is viewed as comprehensive, flexible and meaningful, promotes the adaptation of women who were diagnosed with breast cancer to the problems of disease, so the lower levels of disturbance post-traumatic stress disorder.
\end{abstract}

KEYWORDS: Breast cancer, posttraumatic stress disorder, sense of coherence.

\section{INTRODUÇÃO}

A prevalência das doenças oncológicas é significativa no contexto da saúde das populações, tanto a nível nacional quanto internacional. De acordo com os dados divulgados pelo Instituto Nacional de Estatística (2009) o cancro é a segunda causa de morte em Portugal.

No que diz respeito, especificamente ao cancro da mama tem-se assistido nas últimas décadas a uma diminuição da taxa de mortalidade, contudo a sua incidência tem vindo a aumentar (Costa, 2004)

Verificando-se que apesar da eficácia cada vez maior dos métodos de tratamento do cancro, materializada na sobrevivência crescente. Os doentes continuam a ter que fazer face a uma doença potencialmente debilitante, à dor, à alteração da auto-imagem, à perda de funções fisiológicas e à morte (Andrade, 2000). Logo, a vivência do cancro envolve uma diversidade de stressores que vão desde o diagnóstico, ao tratamento e à sobrevivência a longo prazo (Deep \& Leal, 2000).

Os eventos traumáticos promovem alterações psicológicas significativas nos indivíduos que os vivenciam, podendo acarretar tanto consequências negativas como positivas. À luz desta perspectiva, o diagnóstico de cancro da mama, pode criar uma combinação de formas de stress, que podem conduzir ao aparecimento de sintomas de pós-stress traumático (Andrykowski e col., 1995; Cella \& Tross, 1986; Varela \& Leal, 2008) ou de crescimento pós-traumático (Cordova, Cunningham, Carlson \& Andrykowski, 2001). Encontrando-se ainda por esclarecer quais os factores de vulnerabilidade e resiliência que explicam que a vivência do diagnóstico de cancro seja um fenómeno subjectivo e único.

Um dos factores identificado como determinante na mobilização de respostas adaptativas perante situações extremas é o sentido de coerência. Segundo Antonovsky (1998) o sentido de coerência corresponde a uma disposição e forma vital de ver o mundo, em que este é perspectivado como compreensível, flexível e com significado. Sendo o sentido coerência constituído por três componentes que contribuem de forma complementar para o sentido de coerência, estes são: a capacidade de compreensão,

${ }_{1}$ Professora Assistente, ISEIT/Instituto Piaget/Viseu, margaridavarela@gmail.com

2 Professora Associada, ISPA - Instituto Universitário, ileal@ispa.pt

Submetido em 10-08-2011. Aceite em 18-11-2011.

Citação: Varela, A. M.; Leal, I. (2011). A perturbação de pós-stress traumático e o sentido de coerência em mulheres com cancro da mama. Revista Portuguesa de Enfermagem de

Saúde Mental, 6, 15-19. 
a capacidade de gestão e a capacidade de investimento (Antonovsky, 1998).

Em que a capacidade de compreensão diz respeito à tendência para percepcionar os acontecimentos como ordenados, consistentes, claros, estruturados e explicáveis (Antonovsky, 1993; Nunes, 1999; Pasikowski, Sek \& Scigala, 1994). A capacidade de gestão à apreciação que o indivíduo efectua dos recursos pessoais ou sociais disponíveis para satisfazer as exigências requeridas pelo acontecimento (Antonovsky, 1993; Nunes, 1999; Pasikowski, Sek \& Scigala, 1994) e em que a capacidade de investimento se refere, à crença do indivíduo de que o investimento de energia e interesse na resolução da situação se justifica do ponto de vista emocional e existencial (Antonovsky, 1998; Nunes, 1999).

O sentido de coerência assume desta forma, um carácter marcadamente cognitivo e afectivo, permitindo ao indivíduo estruturar a aparente desordem da sua vida, ou de um determinado acontecimento (Nunes, 1999) ou seja, o sentido de coerência traduz a forma como o indivíduo interpreta os acontecimentos como ordenados e com sentido, o que determina a mobilização de recursos adequados aos acontecimentos com que se depara.

Trata-se de uma variável que determina a capacidade dos indivíduos lidarem com os stressores. Pois, embora seja uma característica própria a todos os indivíduos, é diferente de indivíduo para indivíduo (Nunes, 1999).

A identificação e especificação das características do indivíduo, que facilitam a resolução e a gestão do stress resultante de acontecimentos de vida disruptivos permite compreender os processos que conduzem, por um lado à saúde mental e por outro lado à patologia.

O diagnóstico e tratamentos efectuados para o cancro da mama afectam o bem-estar psíquico, físico e social do indivíduo. A forma como a situação é vivida e o impacto que tem no indivíduo depende das características tanto intrínsecas quanto extrínsecas deste. A identificação e descrição de factores intrínsecos ao indivíduo que contribuem para a compreensão da multiplicidade de respostas exibidas pelos sujeitos, nesta situação específica, tem representado uma área de interesse empírico crescente. Sendo pertinente o estudo do sentido de coerência em doentes com cancro da mama, pois este determina a percepção e o significado que se atribui aos acontecimentos, assim como a resposta do indivíduo a estes (Antonovsky, 1998a).

Consideramos que, os indivíduos que têm elevados níveis de sentido de coerência, perante situações adversas, irão apresentar recursos mais eficazes, logo níveis superiores de adaptação.

Como tal, no presente estudo pretendeu-se analisar se existe uma relação entre o sentido de coerência e o desenvolvimento de perturbação de pós-stress traumático em mulheres a quem foi diagnosticado cancro da mama.

\section{METODOLOGIA}

\section{Tipo de Estudo}

Para alcançar o objectivo acima referido delineou-se um estudo transversal e correlacional.

\section{Participantes}

A população-alvo desta investigação foram mulheres a quem foi diagnosticado cancro da mama. Utilizou-se uma amostra de conveniência, composta por sujeitos disponíveis, cujos critérios de inclusão foram:

1) Diagnóstico de cancro da mama efectuado há mais de um mês;

2) Idade superior a 18 anos;

3) Nível de instrução que lhes permitisse compreender as questões colocadas;

4) Indivíduos que não tivessem sido expostos a nenhum evento stressor capaz de desencadear perturbação de pós-stress traumático, para além do diagnóstico de cancro da mama.

Foram recolhidos ao todo, 89 protocolos de investigação que, após verificados quanto ao preenchimento, tiveram algumas exclusões.

A amostra ficou, então, composta por 84 mulheres a quem foi efectuado o diagnóstico de cancro da mama, que eram seguidas no Hospital de São João situado na cidade do Porto ( $n=25 ; 29,76 \%$ ), na Maternidade Alfredo da Costa situada na cidade de Lisboa $(n=58 ; 69,05 \%)$ e no Centro de Saúde da Cova da Piedade situado no concelho de Almada $(n=1 ; 1,19 \%)$.

Relativamente à idade tratam-se de mulheres com idades compreendidas entre os 18 e os 66 anos, a média das idades foi de 47,86 anos $(d p=9,28)$.

Quanto ao nível de escolaridade, 31 (36,9\%), das mulheres que compuseram a amostra, frequentaram o primeiro ciclo, 9 $(10,7 \%)$ o segundo ciclo, 11 (13,1\%) o terceiro ciclo, $16(19 \%)$ frequentaram o ensino secundário e 17 (20,2\%) completaram o ensino superior/universitário ou politécnico.

Relativamente aos tratamentos efectuados para tratamento do cancro da mama, verificou-se que 38 (45,2\%) ainda estavam a efectuar tratamentos e 46 (54,8\%) já haviam terminado os mesmos. 
Todas as mulheres que compuseram a amostra foram submetidas a cirurgia devido à doença. Quanto ao tipo de cirurgia efectuada, 47 (56\%) realizaram mastectomia a uma das mamas, $35(41,7 \%)$ realizaram cirurgias mais conservadoras (mastectomia parcial ou lumpectomia) e 2 $(2,4 \%)$ realizaram mastectomia a ambas as mamas.

\section{Instrumentos}

a) Questionário de Identificação

Este questionário permitiu a recolha de dados sóciodemográficos e dados clínicos necessários para a selecção e caracterização da amostra. Nomeadamente; permitiu ter acesso a informação relativa à idade, escolaridade e tratamentos efectuados.

Por fim, solicitou-se que os participantes identificassem acontecimentos de vida que tinham tido um impacto negativo. Esta questão permitiu identificar se entre a amostra existiam indivíduos que, para além do diagnóstico de cancro da mama, já haviam experenciado acontecimentos capazes de originar perturbação de pós-stress traumático (American Psychiatric Association, 2002).

b) Questionário Orientação para a Vida

O Questionário Orientação para a Vida é um questionário de auto-resposta composto por 29 itens que permitem avaliar o sentido de coerência, distribuído por três componentes: capacidade de investimento, capacidade de gestão e capacidade de compreensão. Foi aferido para a população portuguesa por Nunes (1999), a partir da escala desenvolvida por Antonovsky (1987).

\section{c) PTSD Checklist Civilian Version}

Trata-se de uma medida auto-administrada, que permite analisar o índice de perturbação de pós-stress traumático em indivíduos expostos a eventos traumáticos de natureza civil. Constituída por 19 itens que remetem para os sintomas de perturbação de pós-stress traumático enunciados no Manual de Diagnóstico e Estatística das Perturbações Mentais.

Posteriormente, a escala foi alterada e adaptada de forma a avaliar a perturbação de pós-stress traumático em indivíduos com cancro, sendo o diagnóstico considerado o evento traumático. Esta adaptação foi efectuada por Cordova, Andrykowski, Kenady, McGrath, Sloan e Redd em 1995.

Para cada um dos itens, os sujeitos assinalam o seu grau de concordância, numa escala de Likert de cinco pontos. A cotação da escala é obtida através do somatório dos itens. Sendo que, a níveis mais elevados está associada uma maior severidade de perturbação de pós-stress traumático. A escala permite ainda, aceder aos sintomas associados a esta perturbação, através da soma dos itens que remetem para cada tipo de sintomatologia.

Os estudos de validação da versão da PCL-C específica para doentes com cancro, efectuados por Cordova e colaboradores (1995; Leiderman-Cerniglia, 2002) revelaram que o instrumento apresenta qualidades psicométricas adequadas.

Dado que, no momento em que foi realizado o presente estudo, não havia sido efectuada nenhuma adaptação para Portugal da versão da PCL-C específica para doentes com cancro, foi necessário proceder à adaptação transcultural da escala. O estudo das características da versão traduzida da PCL-C, permitiu concluir estarmos em presença de uma versão com boas qualidades de fidelidade, consistência interna e validade.

\section{Procedimento}

Após a apresentação dos objectivos do estudo junto dos coordenadores de cada Unidade Médica e obtida a devida autorização para contactar os doentes, procedeu-se ao contacto destes através de carta.

Estes foram informados sobre o objectivo do estudo, a confidencialidade das suas respostas assim como, de estarem a participar num acto voluntário, tendo em consideração as indicações preconizadas pela Associação Médica Mundial na declaração de Helsínquia de 1964 e as emendas referidas na 52. ${ }^{a}$ Assembleia Geral da Associação Médica Mundial (2000).

O protocolo de investigação foi enviado por correio e junto a este seguia um envelope para que fosse possível o retorno do protocolo após o preenchimento. De uma forma geral, verificou-se uma boa adesão dos indivíduos contactados.

De seguida, foi elaborada uma base de dados onde foram introduzidos os dados, estes foram analisados com a ajuda do programa Statistical Package for Social Sciences (SPSS) for Windows, versão 12. Antes de se proceder à análise estatística, foram calculados os valores de cada escala e das subescalas que as constituem, seguindo as instruções e recomendações dos autores das escalas.

\section{Procedimento Estatístico}

Em primeiro lugar foi necessário determinar se a estatística a utilizar era paramétrica ou não-paramétrica. Tendo em consideração que as variáveis em estudo eram ordinais, a estatística adequada seria a não-paramétrica. Porém, de acordo com Pais-Ribeiro (1999) caso se esteja em presença de medidas ordinais de boa qualidade, chega-se aos mesmos resultados quer se utilize estatística paramétrica ou nãoparamétrica. Por outro lado, dado o tamanho da amostra, decidiu-se assumir que esta apresenta uma distribuição próxima da normalidade (Maroco, 2003) desta forma, optouse pela utilização de estatística paramétrica.

Uma vez que se trata de um estudo correlacional foi calculado o coeficiente de correlação de Pearson o que permitiu avaliar 
a intensidade e a direcção da associação. Assinale-se que se consideraram como estatisticamente significativos os testes estatísticos que tiveram articulados um valor de $p \leq 0.05$.

\section{ANÁLISE DOS RESULTADOS}

Ao proceder-se ao estudo das correlações entre o sentido de coerência, os seus componentes e o nível de perturbação de pós-stress traumático relatado pelas mulheres que compunham a amostra, encontraram-se correlações negativas e bastante significativas (Tabela 1).

Os resultados obtidos nesta analise indicaram que quanto maior o sentido de coerência, a capacidade de compreensão, a capacidade de gestão e a capacidade de investimento menor o nível de perturbação de pós-stress traumático relatado pelas mulheres a quem foi diagnosticado cancro da mama.

Quadro 1 - Correlações de Pearson entre sentido de coerência e perturbação de pós-stress traumático $(N=84)$

\begin{tabular}{|l|c|c|c|c|}
\hline & $\begin{array}{c}\text { Sentido } \\
\text { de } \\
\text { coerência }\end{array}$ & $\begin{array}{c}\text { Capacidade } \\
\text { de } \\
\text { compreensão }\end{array}$ & $\begin{array}{c}\text { Capacidade } \\
\text { de } \\
\text { gestão }\end{array}$ & $\begin{array}{c}\text { Capacidade } \\
\text { de } \\
\text { investimento }\end{array}$ \\
\hline P.P.S.T & $-0.47^{* *}$ & $-0.43^{* *}$ & $-0.35^{* *}$ & $-0.50^{* *}$ \\
\hline
\end{tabular}

Legenda: ${ }^{* *} \mathrm{p}<0,01$; P.P.S.T - Perturbação de pós-stress traumático

\section{DISCUSSÃO DOS RESULTADOS}

Um dos factores identificados na literatura como determinante para a mobilização das respostas perante situações extremas é o sentido de coerência. Este corresponde a uma disposição e forma vital de ver o mundo, acreditando que este é compreensível, flexível e que possui um significado (Antonovsky, 1998).

No que tange ao sentido de coerência das mulheres da amostra, os resultados obtidos permitem considerar que apesar da aparente desordem na vida, motivada pelo diagnóstico de uma neoplasia mamária, a capacidade de recuperar a ordem e integrarem o diagnóstico de cancro da mama na sua experiência de vida (Nunes, 1999) se correlaciona negativamente com os níveis de perturbação de pós-stress traumático.

Estes dados vão ao encontro de outros estudos que têm demonstrado o efeito benéfico que o sentido de coerência exerce no bem-estar geral assim como, na saúde física e psíquica dos sujeitos, revelando a sua acção moderadora no desenvolvimento de distúrbios de ansiedade (Vilhena, 2005) e indicando que o sentido de coerência está associado a uma menor vulnerabilidade psicológica.

\section{CONCLUSÃO}

Os resultados obtidos no presente estudo indicam que as mulheres que interpretam o diagnóstico de cancro como um acontecimento ordenado e com sentido apresentam níveis inferiores de pós-stress traumático.

Podemos considerar que o sentido de coerência facilita a mobilização de recursos adequados aos acontecimentos com que estas se irão deparar ao longo do processo de tratamento e recuperação.

Influenciando desta forma, os índices de mortalidade e morbilidade entre as mulheres com cancro da mama. Pois tem sido evidenciado por vários estudos a existência de uma relação entre o sentido de coerência, o bem-estar geral (Vilhena, 2005), a qualidade de vida, a percepção de controlo sobre a doença (Pasikowski, Sek \& Scigala, 1994), os níveis de stress (Gotay, Isaacs \& Pagano, 2004), assim como, a adesão às recomendações e prescrições médicas (Cohen \& Kanter, 2004).

Desta forma, considera-se que o sentido de coerência é uma variável mediadora da percepção de controlo sobre a doença, constituindo-se como um factor que permite a diminuição do sentimento de ameaça e stress vinculado a esta, facilitando a adaptação psicológica, assim como a adopção de comportamentos que auxiliam a recuperação e a reabilitação.

Confrontados com a variabilidade humana, é razoável especular-se sobre a contribuição etiológica desta para determinadas patologias. A constatação efectiva, da existência de uma relação entre variáveis psicológicas e o desenvolvimento de perturbação de pós-stress traumático, contribui para a identificação dos indivíduos que integram o grupo de risco. A identificação das características psicológicas associadas ao desenvolvimento de perturbação de pós-stress traumático, assume desta forma um papel profilático uma vez, que permite identificar os indivíduos em risco e que como tal, podem ser alvo de acções de prevenção ou intervenção, com vista à minimização das repercussões negativas do diagnóstico de cancro da mama.

\section{REFERÊNCIAS BIBLIOGRÁFICAS}

American Psychiatric Association (2002). Manual de diagnóstico e estatística das perturbações mentais $\left(4^{\mathrm{a}} \mathrm{ed}\right.$. Revisão de Texto). Lisboa: Climepsi Editores.

Andrade, T. (2000). Escutar o mar dentro da concha: Descritores do funcionamento psicossomático oncológico. Tese de mestrado em Psicossomática, Instituto Superior de Psicologia Aplicada, Lisboa.

Andrykowski, M., Cordova, M., McGrath, P., Sloan, D. \& Kenady, D. (1995). Stability and change in posttraumatic 
stress disorder symptoms following breast cancer treatment: A 1-year follow-up. Psycho-Oncology, 9 (1), 69-78.

Antonovsky, A. (1987). Unravelling the mystery of health: How people manage stress and stay well. San Francisco: Jossey-Bass, 1987.

Antonovsky, A. (1993). The structure and properties of the sense of coherence scale. Society of Scientific Medicine, 36, (6), 725-733.

Antonosky, A. (1998). The sense of coherence: An historical and future perspective. In H. I. McCubbin, E. A. Thompson, A. I. Thompson, \& J. E. Fromer (Eds.), Stress, coping and health in families: Sense of coherence and resilience (pp. 3-20). London: Sage Publications.

Associação Médica Mundial (2000). Declaration of Helsinki: Ethical principles for medical research involving human subjects. Edinburgh: Author.

Cella, D. \& Tross, S. (1986). Psychological adjustment to survival from Hodgkin's disease. Journal of Consulting and Clinical Psychology, 54, 616-622.

Cohen, M. \& Kanter, Y. (2004). Relation between sense of coherence and glycemic control in type 1 and type 2 diabetes [Abstract]. Behavioral Medicine, 29 (4), 175-183. Retrieved January 20, 2005 from PubMed database.

Cordova, M. Andrykowski, M., Kenady, D. McGrath, P., Sloan, D., \& Redd, W. (1995). Frequency and correlates of posttraumatic stress disorder like symptoms after treatment for breast cancer. Journal of Consulting and Clinical Psychology, 63, 981-986.

Cordova, M., Cunningham, L., Carlson, C. \& Andrykowski. M. (2001). Posttraumatic growth following breast cancer: A controlled comparison study. Health Psychology, 20 (3), 176185.

Costa, L. (2004). O cancro também pode morrer. Porto: Âmbar.
Deep, C. \& Leal, I. (2000). Necessidades e preocupações em doentes oncológicos. Actas do $4 .^{\circ}$ Congresso Nacional de Psicologia da Saúde. Lisboa: ISPA.

Gotay, C. Isaacs, P. \& Pagano, I. (2004). Quality of life in patients who survive a dire prognosis compared to control cancer survivors [Abstract]. Psycho-Oncology, 13 (12), 882 892. Retrieved January 20, 2005 from PubMed database.

Instituto Nacional de Estatística (2009). Óbitos por causa de morte. Consultado em 30 de Junho de 2011 através de http://www.ine.pt

Leiderman-Cerniglia, L. (2002). Psychological factors associated with resistance to PTSD symptoms in women with breast cancer (Doctoral dissertation, Hofstra University, 2001). Dissertation Abstracts International, 62, 10-B.

Maroco, J. (2003). Análise estatística. Com utilização do SPSS. Lisboa: Edições Sílabo.

Nunes, L. A. S. (1999). O sentido de coerência: Operacionalização de um conceito que influencia a saúde mental e a qualidade de vida. Lisboa: Escola Nacional de Saúde Pública.

Pais-Ribeiro, J. L. (1999). Investigação e avaliação em psicologia e saúde. Lisboa: Climepsi Editores.

Pasikowski, T., Sek, H. \& Scigala, I. (1994). Sense of coherence and subjective health concepts. Polish Psychological Bulletin, 25 (1), 15-23.

Varela, M. \& Leal, I. (2008). Perturbação de pós-stress traumático, estratégias de coping e suporte social. In I. Leal, P. Ribeiro, I. Silva \& S. Marques (Org.) Actas do $7^{\circ}$ Congresso Nacional de Psicologia da Saúde (pp. 399-402). Lisboa: Instituto Superior de Psicologia Aplicada.

Vilhena, C. (2005). Resiliência em contexto militar. Tese de mestrado em Consulta Psicológica e familiar, Universidade do Porto, Porto. 